\title{
Treatment of imagined ugliness
}

\section{David Castle \& Tracey Harrison}

The term 'dymorphophobia' - derived from the Greek dysmorphia, meaning ugliness (expressly of the face) - was first coined by Morselli in the late19th century. He described a subjective feeling of a physical defect which the patient feels is noticeable to others, although his appearance is within normal limits. Dysmorphophobia first appeared in the US psychiatric nosology (as an atypical somatoform disorder) in 1980, with the publication of DSM-III (American Psychiatric Association, 1980). However, the term was subsequently criticised both because the condition does not represent a phobia as such, and also because the use of the term had become so broad and imprecise (see Munro \& Stewart, 1991).

Responsive to this imprecision, DSM-III-R (American Psychiatric Association, 1987) scrapped the term 'dymorphophobia', replacing it with 'body dysmorphic disorder' (BDD), a term retained in DSM-IV (American Psychiatric Association, 1994), where again it appears with the somatoform disorders. DSM-IV requires that once the intensity of belief reaches delusional intensity, a separate classification should be made, under delusional disorder, somatic subtype. The World Health Organization (1992), in the Tenth Revision of the International Classification of Diseases (ICD-10), also differentiates between delusional and nondelusional forms of disorder, but has reverted to the older term 'dysmorphophobia'. Thus, 'delusional dysmorphophobia' is subsumed in the category 'persistent delusional disorders'; the symptoms must have been present for at least three months, otherwise the label 'acute and transient psychotic disorder' is deemed more appropriate. On the other hand, 'dysmorphophobia (non-delusional)' is categorised within the somatoform disorders, as a form of hypochondriacal disorder. The DSM-IV diagnostic guidelines for BDD and delusional disorder, somatic subtype, are presented in Boxes $1 \& 2$.

\section{Epidemiology}

There is a paucity of systematic data on the epidemiology of BDD. This relates partly to the fact that individuals with the condition often do not seek professional help, and are reluctant to talk about their problem. Also, clinicians, both psychiatric and non-psychiatric, often simply do not ask the questions required to elicit the symptoms of the disorder.

In the general population, it has been estimated that BDD has a prevalence of around $2 \%$, but in certain groups the prevalence is higher. For example,

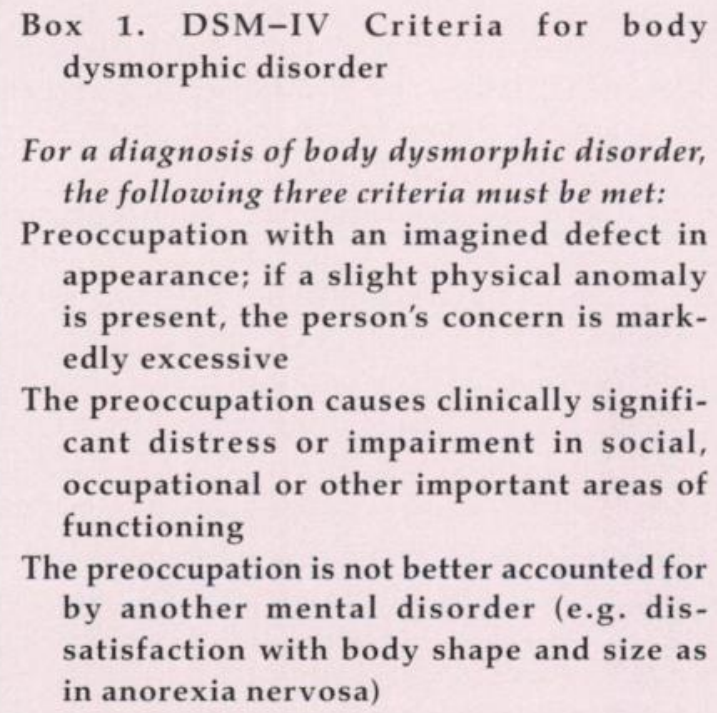

The preoccupation causes clinically significant distress or impairment in social, occupational or other important areas of functioning

The preoccupation is not better accounted for by another mental disorder (e.g. dissatisfaction with body shape and size as in anorexia nervosa)

David Castle is Clinical Director at the Directorate of Mental Health, Fremantle Hospital and Health Service, Fremantle, Western Australia 6160; he has a clinical and research interest in schizophrenia and related disorders, and anxiety disorders, and the overlap between these two groups. Tracey J. Harrison is a clinical psychologist at the Mills Street Centre, 35 Mills Street, Perth, Western Australia 6102; her main clinical interest is in the area of cognitive-behavioural interventions. 
Box 2. Individuals given a diagnosis of body dysmorphic disorder may be given the additional diagnosis of delusional disorder, somatic type if their preoccupation with an imagined defect in appearance is held with delusional intensity

The diagnosis of delusional disorder is given when:

There is the presence of non-bizarre delusions for at least one month duration

Criterion A for schizophrenia has never been met (e.g. hallucinations, disorganised speech, disorganised or catatonic behaviour, negative symptoms); tactile or olfactory hallucinations may be present in delusional disorder if they are related to the delusional theme

Apart form the impact of the delusion or its ramifications, functioning is not markedly impaired and behaviour is not obviously odd or bizarre

If mood episodes have occurred concurrently with delusions, their total duration has been brief relative to the duration of the delusional disorder

The disturbance is not due to the direct physiological effects of a substance or a general medical condition

rates of around $5 \%$ have been found in individuals seeking medical treatment. In those seeking cosmetic surgery, rates are even higher, although there are few methodologically sound studies of such populations, and diagnostic criteria have often been rather loose. Hay (1970) found that $16(35 \%)$ of 45 patients requesting cosmetic rhinoplasty had 'severe neurosis', and one had a psychotic disorder. Connolly \& Gibson (1978) performed a follow-up of 86 patients who underwent rhinoplasty for cosmetic reasons, and compared them with 101 patients whose rhinoplasty had been occasioned by disease or injury. Of the 'cosmetic' group, 32 (37\%) were considered at follow-up to have 'severe neurosis', and a further six (7\%) manifested schizophrenia; this is in marked contrast to nine $(9 \%)$ and one $(1 \%)$ of the 'non-cosmetic' group, respectively.

In psychiatric populations, rates of BDD are also elevated. For example, Phillips et al (1996), in a study of 80 out-patients with depression, identified $8 \%$ as meeting criteria for BDD. Using a different approach, Oosthuizen et al (1998) used a self-report measure tapping over-concern with physical appearance, in 63 psychiatric in-patients (most had schizophrenia or severe mood disorders); they reported $35 \%$ of the patients as having excessive bodily concern, but do not state how many would have met criteria for BDD.

Body dysmorphic disorder usually begins in adolescence, with a mean onset of illness around the age of 16 years. There is often a considerable lag before the disorder comes to the attention of health professionals. The male:female ratio is roughly equal, in most series.

\section{Imagined ugliness as a symptom}

While Morselli's notion of 'dysmorphophobia' has entered the modern psychiatric nosology, as outlined above, it is as well to remember that a view of the self as unattractive, or even ugly, can occur to a greater or lesser extent in other psychiatric disorders. Indeed, it has been suggested that the term 'dysmorphic concern' might better describe this symptom, as it does not imply any specific diagnosis (Oosthuizen et al, 1998). After all, some degree of concern about physical appearance is common in the general population, as documented in a survey of students in the USA (Fitts et al, 1989), 70\% of whom expressed some dissatisfaction about their looks; indeed, $48 \%$ described a degree of preoccupation with some aspect of their physical appearance.

Dysmorphic concern can also be a symptom of a number of underlying psychiatric disorders, and it is always important to exclude such disorders in anyone presenting with an apparent over-concern about physical appearance. A number of pointers are given in Box 3 to aid the clinician in this regard.

\section{Psychotic disorders}

Complaints often have a bizarreness about them, for example, that one side of the face has been altered in some way, or that internal organs have been rotated. There may also be a delusional explanation, for example, that God did this as a sign to the patient that he is chosen, or the Messiah; or that aliens performed surgery by means of laser beams, leaving no external scars.

\section{Depression}

Over-concern about appearance is relatively common in depression, and can be seen as one of the cognitive distortions associated with low mood. Usually the patient will complain of thinking that he or she is looking old and unattractive, but in 
Box 3. Clinical pointers to 'secondary' dysmorphic concern

Psychotic disorders: be alerted if there are other symptoms of psychosis, e.g. disturbance of affect, or formal thought disorder; ask about the patient's explanation for the 'defect' in appearance

Depression: be alerted if other features of depression, and ask about mood, including vegetative symptoms, etc.; attribution might be that this is punishment, or deserved in some way

Obsessive-compulsive disorder: ask for other features of the disorder, including other obsessional thoughts, other compulsive acts

Social phobia: elicit the core cognition which leads to feeling uncomfortable in social situations (i.e. fear of negative evaluation); establish behavioural consequences, notably avoidance of social situations

Somatoform disorders: elicit other features of this group of disorders, including fear of ill health (hypochondriasis) and multiple somatic complaints (somatisation disorder)

Anorexia nervosa: be alerted in young women with overconcern about body shape and size, and who are underweight; ask about dieting, bingeing, purging, excessive exercise, etc.

Delusional disorder: nominally, once the dysmorphic concern reaches delusional intensity, the diagnosis should, according to DSM-IV, be in the psychotic category

extreme cases, the patient might believe that he or she is actually decaying, or rotting away (e.g. as in Cotard's syndrome).

\section{Obsessive-compulsive disorder}

In many cases, the phenomenology displayed by individuals with an excessive degree of concern about their physical appearance, shows similarities with that displayed by patients with obsessivecompulsive disorder (OCD). For example, the thoughts of ugliness can be experienced as ruminations; that is, thoughts recognised as the person's own, but which are intrusive, ego dystonic, and not readily dismissed from the mind. The intrusive thoughts can lead the sufferer to check the mirror repeatedly, to ask for reassurance from others about their appearance, or to resort to ritualistic 'disguising' of the offending physical feature, through use of make-up, for example. Some patients also exhibit elements of habit disorder, with the compulsion to pick at the skin, or pluck eyebrows, etc. These exercises can take up many hours of the person's day. Indeed, some commentators believe that BDD is part of an 'obsessive-compulsive spectrum' (see below).

\section{Social phobia}

The fear of negative evaluation by others, which is the hallmark of social phobia, can also be a part of dysmorphic concern. Sometimes the prominent cognition is that others will cast judgment about the way the individual looks, and this might lead to anxiety in social situations, and avoidance thereof.

\section{Somatoform disorders}

Here the main concern is related to bodily ill health or malfunction, with consequent help-seeking behaviour. This group of disorders encompasses somatisation disorder, hypochondriasis and somatoform pain disorder. The DSM-IV classifies body dysmorphic disorder in this group, but it sits uncomfortably here, and is probably more usefully considered along with the anxiety disorders, notably OCD, as alluded to above.

\section{Anorexia nervosa}

This is a specific exclusion for a diagnosis of body dysmorphic disorder in DSM-IV, but it is obvious that the central cognition associated with this disorder is compatible with the label 'dysmorphic concern', in that there are intrusive thoughts about bodily appearance, which might be particularly focused on certain body parts, for example, the thighs or waist. Of course, treatment in such cases must address the eating disorder itself, rather than focus on the distorted body image, and it is this fact that makes it sensible to distinguish anorexia nervosa from body dysmorphic disorder.

\section{Personality disorder}

It should be noted that disordered self-image can also be seen in individuals with certain personality structures. In particular, 'marked and persistent identity disturbance' with uncertainty about selfimage, is one of the criteria for borderline personality disorder. 


\section{Comorbidity}

Rates of comorbidity among patients with BDD are high. The most consistent findings have been of comorbidity with depressive disorders (expressly 'atypical' depression, OCD and social phobia). For example, in a survey of 130 cases of BDD, Phillips et al (1995) reported lifetime prevalence rates of comorbid depression of $83 \%$, while rates of OCD and social phobia were $29 \%$ and $35 \%$, respectively. Of course, it is difficult to tease apart cause and effect in these scenarios, in that the intrusion on rolefunctioning seen in patients with BDD can result in depression and fear of negative evaluation of appearance by others, which might mimic social phobia and result in avoidance of social situations. Similarly, the symptoms of BDD show many similarities with those of $O C D$, as detailed below.

\section{A variant of $\mathrm{OCD}$ ?}

As outlined above, the classification of BDD in DSMIV, where it is presumed to be a discrete entity and where it is classified with the somatoform disorders, has been challenged. A number of commentators, notably Phillips et al (1995), have pointed out the low rates of comorbidity with other somatoform disorders, the lack of familial aggregation with somatoform disorders, and the therapeutic response to selective serotonin reuptake inhibitors (SSRIs) (see below) as evidence of the inappropriateness of this classification.

Thus, these authors have suggested that BDD is more appropriately grouped with $O C D$, as part of an 'OCD spectrum' which encompasses OCD, atypical depression, eating disorders, hypochondriasis and impulse control disorders. The clinical features of BDD, and the overlap with the symptomatology of these disorders, have been alluded to above, as has the high rate of comorbidity between OCD and BDD. Adding further weight to this argument is the finding of familial aggregation of BDD with OCD; for example, Hollander et al (1993) reported $17 \%$ of the first-degree relatives of their group of BDD patients to have OCD, although the fact that $78 \%$ of the BDD patients were comorbid for OCD might well have inflated this estimate. Also, there are demographic similarities between the two disorders, notably onset in late adolescence and early adulthood, an equal gender ratio, and similar longitudinal course. But more important is the relative specificity of response of BDD to treatments usually found effective for OCD, notably antidepressants with predominantly serotonergic activity, and the behavioural intervention of exposure and response prevention (see below).

\section{Is there a delusional subtype of BDD?}

Convention, and DSM-IV, have dictated that it is important to distinguish between delusional and non-delusional forms of BDD. Indeed, cases in which the dysmorphic concern reaches delusional intensity are required by DSM-IV to be classified with the delusional (paranoid) disorders, and Munro (1980) suggests that such patients have a form of monosymptomatic hypochondriacal delusion which warrants treatment with the antipsychotic, pimozide. However, such a dichotomy on the basis of intensity of belief has been challenged, and after all, it has now been acknowledged in DSM-IV that the compulsions associated with OCD can reach delusional intensity, without the necessity for a change of diagnosis.

Phillips et al (1994) investigated 100 cases of BDD specifically to address the issue of whether there is a delusional subtype of the disorder. These authors found that 52 BDD cases with the 'delusional' form of disorder did not differ from their 'non-delusional' counterparts in terms of demographic parameters, phenomenology (apart from intensity of belief), associated features or comorbidity. Furthermore, neither group responded well to treatment with antipsychotic medications, while therapeutic success was found with serotonergic antidepressants. However, the 'delusional' patients did rate higher on a modified form of the Yale-Brown Obsessive-Compulsive Scale (Goodman et al, 1989), suggesting that they merely had a relatively severe form of the same disorder.

\section{Treatment of BDD}

As detailed above, the symptom of imagined ugliness can be seen in a number of psychiatric conditions, and before treatment is embarked upon, it is important to ascertain whether it is simply a manifestation of, for example, depression or schizophrenia, in which case the condition would require treatment in its own right. Furthermore, depressive and anxiety comorbidity in true 'primary' BDD is common, and again needs to be addressed 
as part of a comprehensive treatment plan. Henceforward, then, we will deal only with 'primary' BDD, where the vast majority of treatment trials have been in the realm of psychotropic drugs and/or psychological treatments. A handful of studies have investigated the role of electroconvulsive therapy (ECT), and a small number of reports have addressed the efficacy of plastic surgery or dermatological treatments for BDD.

\section{Plastic surgery and dermatological treatments}

One's instinctive belief would be that surgery is contraindicated in the treatment of BDD, in that the underlying cause is not addressed, and the expense and discomfort of surgery might leave the patient dissatisfied and potentially litigious. Hay (1970) suggested that those patients with a 'normal personality' could benefit from cosmetic surgery, but that 'neurotic' patients would not benefit. He provided no follow-up data to test this theory, but most available evidence suggests that surgery or dermatological intervention are unlikely to help BDD patients. For example, Phillips et al (1995) found that of 43 BDD patients who underwent an array of dermatological treatments including antibiotics, steroids and dermabrasion, only five (11\%) considered the treatment to have been successful. In the review of Phillips et al (1993), eight of 30 patients $(27 \%)$ had undergone surgical procedures; only one of the 25 procedures was felt by the patient to have been helpful.

A small proportion of BDD sufferers with minor deformities do apparently show at least some benefit from surgical or dermatological treatment (Phillips et al, 1995). One could argue that such individuals did not have BDD at all, and that patients with 'true' BDD will, by definition, not benefit from such interventions. However, this argument can get bogged down in semantics, and a more useful approach might be to screen individuals requesting plastic surgery for psychiatric comorbidity. However, many patients who do have psychological problems underlying their dysmorphic concern, refuse psychiatric consultation (Fukuda, 1977). Having said this, it is incumbent upon practitioners in those 'cosmetic' specialities to be aware of the potential for underlying psychiatric disorders, and to seek a psychiatric opinion if they are concerned in this regard.

\section{Electroconvulsive therapy}

We are aware of no systematic assessments of the efficacy of electroconvulsive therapy (ECT) for BDD.
There have been isolated reports of BDD patients who did benefit from ECT, but most investigators have generally found it to be unsuccessful. For example, Phillips et al (1993) reported ECT to have benefited none of their four BDD patients who had been treated with it. A potentially interesting issue which has not been adequately explored is whether depressed patients with overvalued ideas or delusional beliefs about their bodies would respond particularly favourably to ECT, given the suggestion that mood-congruent psychotic beliefs predict response to ECT in depressed patients.

\section{Medication}

Most medication trials in BDD have been single case studies or small series. Diagnostic criteria have seldom been operationalised, and there has been significant psychiatric comorbidity. Studies have been open and non-randomised. Some have included a psychological intervention and thus are not 'pure' pharmacological trials. There is also the problem of publication bias, with effective treatments being more likely to be publicised than failures. A number of reported series have overlapping samples, making assessment of rates of response difficult. Also, the majority of reports have been from a handful of investigators, mostly with a particular interest in $B D D$, suggesting potential referral bias.

Given these methodological limitations, it is clear that the conclusions cannot be definitive, but some trends can be delineated, at least regarding two main classes of drugs, namely neuroleptics and antidepressants. Anxiolytic agents have not been rigorously explored in sufficient numbers of patients to allow any meaningful conclusions to be drawn.

\section{Neuroleptics}

Given the debate regarding the phenomenology of BDD, and the fact that the intensity of belief can sometimes reach delusional proportions (as outlined above), it is important to consider the efficacy of neuroleptic medication in afflicted individuals. Open trials of neuroleptics have been reported by a number of different investigators, with most showing little or no benefit. An important consideration is whether there is a differential response to medication between subjects with delusional and non-delusional forms of BDD. McElroy et al (1993) reported little benefit from antipsychotic medication in deluded or non-deluded BDD patients. Phillips et al (1994) compared response to a number of agents in deluded and nondeluded subjects, revealing only one of 73 trials of neuroleptics to have been effective; intriguingly, the 
most effective agents in both deluded and nondeluded patients were SSRIs.

The lack of efficacy of neuroleptics in the treatment of BDD, irrespective of whether the intensity of belief is gauged to be 'delusional', suggests a mechanism of illness distinct from the often phenomenologically related 'monosymptomatic hypochondriacal delusions' reported to be peculiarly responsive to the neuroleptic, pimozide (Munro, 1980). Some authors believe that some patients with delusional overconcern about their physical appearance have a form of paranoia, responsive to pimozide, and that those who do not respond to this agent have a neurotic disorder rooted in personality. Such a distinction invokes a 'therapeutic test' to aid diagnosis, and is not supported by the evidence reviewed here. Readers should also be aware of the necessity for cardiac monitoring with pimozide.

\section{Antidepressants}

Regarding antidepressants, the most consistent clinical benefit reported has been with serotonergic agents (clomipramine, SSRIs), though the degree of response has varied, and some patients have failed on such agents. Tricyclic antidepressants apart from clomipramine have generally not been useful. Only the large series of McElroy and colleagues and of Phillips and colleagues have sufficient numbers to gauge in any meaningful way the proportion of patients who respond to SSRIs or clomipramine. Those series suggest rates of the order of $50 \%$ for SSRIs, and 70\% for clomipramine. Effective doses of medication have usually been high (up to $200 \mathrm{mg}$ sertraline, $80 \mathrm{mg}$ fluoxetine and $300 \mathrm{mg}$ clomipramine daily), but some patients have responded to more modest doses.

A number of studies have suggested efficacy for monoamine oxidase inhibitors (MAOIs) in BDD. For example, Jenicke (1984) described the "complete response" to tranylcypramine of a patient who had failed to respond to neuroleptic or heterocyclic antidepressant medications, and Barr et al (1992) described a patient whose BDD symptoms, but not depression, responded to a MAOI. However, a number of authors have reported failed trials of MAOIs in BDD. In their review, Phillips et al (1994) found a success rate of $29 \%$ for MAOIs in BDD. There is no clear indiction of which clinical parameters predict a response to MAOIs, but it appears that both deluded and non-deluded subjects might benefit.

It will be noted that for many BDD patients there is depressive comorbidity, and it is possible that the improvement in BDD symptoms might be consequent upon an improvement in mood. However, some patients who were not clinically depressed did respond to serotonergic agents, and the relative specificity of serotonergic agents among the antidepressants suggests a mechanism apart from purely improvement in mood.

\section{Psychological interventions}

There is a lack of empirical research regarding the effectiveness of psychological interventions for BDD. In those studies which have been reported, diagnosis has often been unsystematic, with many individuals being diagnosed with comorbid disorders, such as obsessive-compulsive disorder, depression and social phobia. Often, individuals have commenced using psychological interventions while also using pharmacological agents. Furthermore, descriptions of what actually took place within therapy are often vague, and duration of intervention varies greatly between studies. These factors make replication difficult.

There have been single case reports regarding the use of psychodynamic and psychoanalytically oriented therapy (Bloch \& Glue, 1988; Philippopoulos, 1979). Duration of therapy has varied from 12 to 24 months, with anything from weekly to thriceweekly sessions. The main aim of treatment appears to be resolution of underlying psychic conflicts. While reportedly successful, these few uncontrolled studies, with no objective measurement of symptomatology or outcome, cannot be considered in any way definitive.

The remaining research regarding psychological treatment for BDD focuses on variations of cognitive and behavioural therapies, with a number of case reports and small case series having been reported (see Box 4). In terms of behavioural interventions, Gomez-Perez et al (1994) reviewed 30 patients with BDD who were treated with exposure and response prevention mainly targetting social avoidance situations. Most patients showed improvement in terms of avoidance behaviour, work and social adjustment, and intensity of beliefs. We turn now to those few controlled studies which have assessed

\footnotetext{
Box 4. Elements of behavioural and cognitive-behavioural therapy for body dysmorphic disorder
}

Cognitive therapy involving modification of intrusive thoughts of body dissatisfaction and overvalued ideas about physical appearance

Exposure to avoided body image situations

Response prevention to eliminate body checking 
the efficacy of cognitive-behavioural therapy (CBT) in disorders of body image. Rosen et al $(1989,1990)$ randomly assigned college students who were assessed as having disturbed body image to a CBT group or to a minimal treatment group. Cognitivebehavioural therapy was conducted in small groups over a six-week period. Therapy consisted of correction of size and weight overestimation, modification of distorted and negative thoughts, and exposure to anxiety-provoking situations. Greater improvements in size overestimation, body dissatisfaction and behavioural avoidance were found in the CBT group than the minimal treatment group.

In a controlled study of treatment effects for BDD using cognitive and behavioural therapy, Rosen $e t$ al (1995) randomly assigned 54 women diagnosed with BDD to group-based CBT or a no-treatment group. Therapy involved modification of intrusive thoughts of body dissatisfaction and overvalued ideas about physical appearance, exposure to avoided body image situations, and elimination of body-checking. Symptoms of BDD were significantly reduced within the therapy group $(82 \%$ posttreatment and $77 \%$ at follow-up no longer met criteria for BDD), and measures of psychological symptoms and self-esteem also improved in therapy subjects.

\section{Conclusions}

Imagined ugliness might be considered a symptom of a number of psychiatric disorders, but sometimes presents as a primary disorder, in which case a diagnosis of dysmorphophobia or BDD, should be considered. As such, BDD does not sit comfortably with the other somatoform disorders, and should probably be considered as being related to OCD. The distinction of delusional from non-delusional forms does not appear therapeutically informative. Treatment of any primary or comorbid condition is necessary, but in primary BDD the most effective therapeutic options appear to be serotonergic antidepressants (and perhaps MAOIs in those who fail such treatment), and some form of behavioural or cognitive-behavioural therapy. More research is required to determine whether these interventions are equally efficacious, and whether they might be mutually enhancing.

\section{References}

American Psychiatric Association (1980) Diagnostic and Statistical Manual of Mental Disorders (3rd edn) (DSM-III). Washington, DC: APA.
- (1987) Diagnostic and Statistical Manual of Mental Disorders (3rd edn, revised) (DSM-III-R). Washington, DC: APA.

- (1994) Diagnostic And Statistical Manual of Mental Disorders (4th edn) (DSM-IV). Washington, DC: APA.

Barr, L.C., Goodman, W. K. \& Price, L. H. (1992) Acute exacerbation of body dysmorphic disorder during tryptophan depletion. American Journal of Psychiatry, 149, 1406.

Bloch, S. \& Glue, P. (1988) Psychotherapy and dysmorphobia: A case report. British Journal Of Psychiatry 152, 271-274.

Connolly, F. H. \& Gibson, M. (1978) Dysmorphophobia - a long term study. British Journal Of Psychiatry, 132, 568570 .

Fitts, S. N., Gibson P., Redding , C. A., et al (1989) Body dysmorphic disorder: Implications for its validity as a DSM-III-R clinical syndrome. Psychological Reports, 64, 655-658

Fukuda, O. (1977) Statistical analysis of dysmorphophobia in out-patient clinic. Japanese Journal of Plastic and Reconstructive Surgery, 20, 569-577

Gomez-Perez, J. C., Marks, I. M. \& Gutierrez-Fisal, J. L. (1994) Dysmorphophobia: clinical features and outcome with behaviour therapy. European Psychiatry, 9, 229-235.

Goodman, W. K., Price, L. H., Rasmussen, S. A., et al (1989) The Yale-Brown Obsessive-Compulsive Scale. Archives of General Psychiatry, 46, 1006-1016.

Hay, G.G. (1970) Dysmorphophobia. British Journal of Psychiatry, 116, 399-406.

Hollander, E., Cohen, L. J. \& Simeon, D. (1993) Body dysmorphic disorder. Psychiatric Annals, 23, 359-364.

Jenicke, M. A. (1984) A case report of successful treatment of dysmorphophobia with tranylcypromine. American Journal of Psychiatry, 141, 1463-1464.

McElroy, S. L., Phillips, K. A., Keck, P. E., et al (1993) Body dysmorphic disorder: Does it have a psychotic subtype? Journal of Clinical Psychiatry, 54, 389-395

Munro, A. (1980) Monosymptomatic hypochondriacal psychosis. British Journal of Hospital Medicine, 24, 34-38.

- \& Stewart, M. (1991) Body dysmorphic disorder and the DSM-IV: the demise of dysmorphophobia. Canadian Journal of Psychiatry, 36, 91-96.

Oosthuizen, P., Lambert, T. \& Castle, D. J. (1998) Dysmorphic concern: prevalence and associations with clinical variables. Australia and New Zealand Journal of Psychiatry, 32, 129-132

Philippopoulos, G. S. (1979) The analysis of a case of dysmorphophobia. Canadian Journal of Psychiatry, 24, 397401.

Phillips, K. A., McElroy, S. L., Keck, P. E. Jr, et al (1993) Body dysmorphic disorder: 30 cases of imagined ugliness. American Journal Of Psychiatry, 150, 302-308.

-, - - - et al (1994) A comparison of delusional and nondelusional body dysmorphic disorder in 100 cases. Psychopharmacology Bulletin, 30, 179-186.

- - Hudson, J. I., et al (1995) Body dysmorphic disorder: An obsessive-compulsive sectrum disorder, a form of affective spectrum disorder, or both? Journal of Clinical Psychiatry, 56 (suppl. 4), 41-51.

-, Nierenberg, A. A., Brendel, G., et al (1996) Prevalence and clinical features of body dysmorphic disorder in atypical major depression. Journal of Nervous and Mental Disease, 184, 125-129.

Rosen, J. C., Saltzberg, E. \& Srebnik, D. (1989) Cognitive behaviour therapy for negative body image. Behaviour Therapy, 20, 393-404.

- Cado, S., Silberg., N. T., Srebnik, D., et al (1990) Cognitive-behaviour therapy with and without size perception training for women with body image disturbance. Behaviour Therapy, 21, 481-498.

-, Reiter, J. \& Orosan, P. (1995) Cognitive-behavioural body image therapy for body dysmorphic disorder. Journal of Consulting And Clinical Psychology, 63, 263-269.

World Health Organization (1992) The Tenth Revision of the International Classification of Diseases and Related Health Problems (ICD-10). Geneva: WHO. 


\section{Multiple choice questions}

1. Over-concern with physical appearance may be a symptom of:
a body dysmorphic disorder
b anorexia nervosa
c depression
d schizophrenia
e borderline personality disorder.

2. 'Delusional' body dysmorphic disorder:

$a$ is categorised in DSM-IV with the psychotic disorders

b is phenomenologically quite distinct from the non-delusional form

c responds well to antipsychotic medication

d often responds to SSRIs

e might be considered a severe form of nondelusional body dysmorphic disorder.

3. Body dysmorphic disorder and obsessivecompulsive disorder:

a show many phenomenological similarities

b have distinct underlying pathogenic mechanisms

c both respond to exposure/response prevention treatment

d both respond to SSRIs

e there is no familial aggregation of OCD cases in individuals with BDD.
4. Regarding treatments for BDD:

a psychodynamic psychotherapy is the most successful treatment

b exposure/response prevention is successful in a proportion of cases

c cognitive therapy has no place in treatment

d treatment requires antipsychotics if beliefs are held with delusional intensity

e MAOIs might be successful where other treatments have failed.

5. Regarding epidemiology of BDD:

a rates are elevated in those seeking plastic surgery

b BDD usually begins abruptly in adolescence

c girls are more commonly affected than boys

d BDD is usually short-lived

e the diagnosis of BDD cannot be made in individuals with anorexia nervosa.

MCQ answers

$\begin{array}{llllllllll}\text { 1 } & & \text { 2 } & & \text { 3 } & & \text { 4 } & & \text { 5 } \\ \text { a } & \text { T } & \text { a } & \text { T } & \text { a } & \text { T } & \text { a } & \text { F } & \text { a } & \text { T } \\ \text { b } & \text { T } & \text { b } & \text { F } & \text { b } & \text { F } & \text { b } & \text { T } & \text { b } & \text { F } \\ \text { c } & \text { T } & \text { c } & \text { F } & \text { c } & \text { T } & \text { c } & \text { F } & \text { c } & \text { F } \\ \text { d } & \text { T } & \text { d } & \text { T } & \text { d } & \text { T } & \text { d } & \text { F } & \text { d } & \text { F } \\ \text { e } & \text { T } & \text { e } & \text { T } & \text { e } & \text { F } & \text { e } & \text { T } & \text { e } & \text { F }\end{array}$

\section{Commentary}

\section{Isaac Marks}

As Castle \& Harrison note in their fine review, imagined ugliness is a not uncommon problem presenting to psychiatrists. The "considerable lag before the disorder comes to the attention of health professionals" was a mean of seven years in the 30 cases of Gomez-Perez et al (1994). Whether the problem is called dysmorphophobia, body dysmorphic disorder (BDD) or something else, its coherence of features resembles that of other psychiatric syndromes.
Diagnostic systems dwell on one's belief that one looks ugly, but an otherwise similar clinical picture is seen in many patients who believe that they smell bad (Marks, 1987, p. 371; Gomez-Perez, et al 1994). Like other patients with dysmorphophobia (cases of BDD), those who complain that they smell may have surgery to remove axillary glands, avoid social contact, conceal the perceived problem (by washing a lot and using vast amounts of deodorants) and may improve with treatment by exposure, and 